\title{
AVALIAÇÃO DO ESCORE HOSPITAL COMO PREDITOR DE MORTALIDADE E REINTERNAÇÃO EM PACIENTES ADMITIDOS EM ENFERMARIA CLÍNICA EM UM HOSPITAL UNIVERSITÁRIO
}

William Damian Perdonsini Klein ${ }^{1}$; Vander José Dall' Aqua da Rosa'; Carolina Perez Moreira'; Verônica Westphal'; Diego da Rosa Miltersteiner. $^{2}$

\section{Resumo}

Introdução: O escore HOSPITAL, capaz de identificar potenciais pacientes com risco de reinternações precoces, é composto por sete variáveis independentes e classifica os pacientes em baixo, médio e alto risco (Donzé, 2013; Allaudeen, 2011). A pontuação obtida está relacionada diretamente com a chance de reinternar em 30 dias (Donzé, 2013). Metodologia: O presente estudo testou o escore HOSPITAL como preditor de mortalidade e reinternação nos pacientes de um hospital universitário do sul do Brasil. Estudo de coorte retrospectivo. A amostra do estudo foi consecutiva, abrangendo todos os pacientes clínicos adultos internados entre 01 de maio de 2013 a 28 de fevereiro de 2014. De forma retrospectiva, foram analisados sódio e hemoglobina antes da alta, neoplasia, internação eletiva, número de admissões hospitalares no último ano, tempo de internação e realização de procedimento invasivo dos prontuários de 2494 pacientes, sendo incluídos no estudo 372. Os dados foram transcritos por dupla digitação em planilha do Excel e analisados pelo método qui-quadrado utilizando o programa Prism 6. Resultados: Dos 372 pacientes estudados, a taxa de mortalidade total em até 90 dias após a

\footnotetext{
1 Graduandos na faculdade de medicina da Universidade Luterana do Brasil Canoas, RS

2 Médico nefrologista e intensivista, docente na Universidade Luterana do Brasil - Canoas, RS
} 
alta hospitalar foi de 11,2\% e a de reinternação em 30 e 90 dias foi 10,75\% e 23,12\%, respectivamente. Em relação à análise do escore HOSPITAL, os dados de mortalidade por escore (alto, médio e baixo risco), não se conseguiu diferenciar de maneira estatisticamente significativa os pacientes com maior risco de mortalidade. Por outro lado, o escore pode diferenciar de maneira estatisticamente significativa, comparável aos dados da literatura, a chance do paciente necessitar de reinternação em 30 dias ( $\mathrm{P}<0.0005, \mathrm{RR}=1.86$ [IC 95\% 1.082 - 3.209]), comparando pacientes de baixo risco com os de alto risco. Conclusão: Mortes e readmissões hospitalares são onerosas para o sistema de saúde e resultam em impacto emocional importante. Apesar do escore HOSPITAL não apresentar relação como preditor de mortalidade após a alta hospitalar, o mesmo demonstrou ser uma ferramenta de fácil aplicação com validade estatística significativa para identificar os pacientes mais susceptíveis a reinternarem precocemente. Com isso, torna-se possível instituir medidas de acompanhamento após alta e concentrar recursos para realizar intervenções de cuidados de transição mais intensas para os pacientes de alto risco capazes de reduzir, no montante final, os gastos com readmissões (Metzger, 2012; Bendassat, 2000; Umesh, 2012).

Palavras Chave: reinternação precoce; alta hospitalar; morbimortalidade 


\section{Tabela e figuras}

Figura/Tabela 1: Reinternação em 30 dias

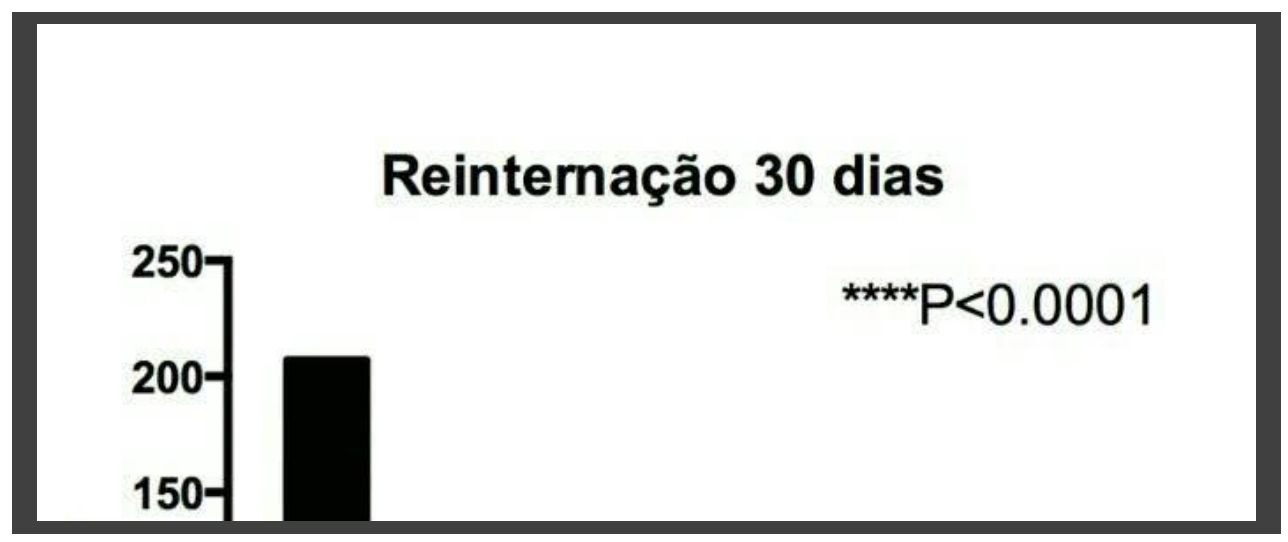

\section{Avaluation of HOSPITAL score as predictor of mortality and readmission to patients of an Universitary Hospital}

\section{Abstract}

Hospital score is capable of identifying potential risk of early readmission. It's composed by seven independente variables and cassifies patients by low, mild and high risk of readmission. This study is a retrospective cohort. We've tested the HOSPITAL score as predictor of mortality and readmission in patients of an universitary hospital in South of Brazil. Our sample of 372 patients was collected between May 1rst, 2013 and February 28th, 2014. Mortality rate in those patients after 90 days was 11,2\%, and readmission rate after 30 and 90 days were $10,75 \%$ and 23,12\%. Our 
conclusion is thar death and readmission results in great impacts for health system and also as an emotional impact.

Keywords: early readmission. discharge. morbimortality 


\section{Referências}

Donzé J, Aujesky D, Williams D, Schnipper JL. Potentially avoidable 30-day hospital readmissions in medical patients: derivation and validation of a prediction model. JAMA Intern Med. 2013; 173:632-638.

Allaudeen N, Vidyarthi A, Maselli J, Auerbach A. Redefining readmission risk factors for general medicine patients. J Hosp Med. 2011; 6:54-60.

Metzger J, Lorincz C. Preventing Hospital Readmissions: The first test case for continuity of care. Global Institute for Emerging Healthcare Practices. July 2012.

Brasil. Ministério da Saúde, ConselhoNacional das SecretariasMunicipais de Saúde. O SUS de A aZ :garantindosaúdenosmunicípios. 3.ed. Brasília: Editora do Ministério da Saúde, 2009. 480 p.

Bendassat J, Taragin M. Hospital readmissions as a measure of quality of health care: advatagens and limitations. Arch Intern Med. 2000 Apr; 160(8):1074-81

Umesh N, Khot. Exploring the Risk of Unintended Consequences of Quality Improvement Efforts. J Am Coll of Cardiol 2012;60:813-2. 